\title{
UCRL-CONF-205469
}

LAWRENCE LIVERMORE N A TIO N A L

\section{Self Organization of Wireless Sensor Networks Using Ultra-Wideband Radios}

F. Nekoogar, F. Dowla, A. Spiridon

July 23, 2004

Self Organization of Wireless Sensor Networks Using Ultra-Wideband Radios Atlanta, GA, United States September 19, 2004 through September 22, 2004 
This document was prepared as an account of work sponsored by an agency of the United States Government. Neither the United States Government nor the University of California nor any of their employees, makes any warranty, express or implied, or assumes any legal liability or responsibility for the accuracy, completeness, or usefulness of any information, apparatus, product, or process disclosed, or represents that its use would not infringe privately owned rights. Reference herein to any specific commercial product, process, or service by trade name, trademark, manufacturer, or otherwise, does not necessarily constitute or imply its endorsement, recommendation, or favoring by the United States Government or the University of California. The views and opinions of authors expressed herein do not necessarily state or reflect those of the United States Government or the University of California, and shall not be used for advertising or product endorsement purposes. 


\title{
Self Organization of Wireless Sensor Networks Using Ultra- Wideband Radios
}

\author{
Faranak Nekoogar, Farid Dowla, Alex Spiridon \\ Lawrence Livermore National Laboratory, Livermore, CA 94550 USA
}

\begin{abstract}
Ultra-wideband (UWB) technology has proven to be useful in short range, high data rate, robust, and low power communications. These features can make UWB systems ideal candidates for reliable data communications between nodes of a wireless sensor network (WSN). However, the low powered UWB pulses can be significantly degraded by channel noise, inter-node interference, and intentional jamming. In this paper we present a novel interference suppression technique for UWB based WSNs that promises self-organization in terms of power conservation, scalability, and channel estimation for the entire distributed network.
\end{abstract}

Index Terms - Channel estimation, interference mitigation, jitter, self-organization, synchronization, ultrawideband, wireless sensor networks.

\section{INTRODUCTION}

Wireless sensor networks (WSNs) have become increasingly popular in military and civilian applications such as surveillance, monitoring, disaster recovery, home automation and many others. In a wireless sensor network, a large population of sensor nodes are scattered in an inaccessible area to process and transfer data to the expected destination. The individual nodes in these networks should have small form factor, light weight, provide long service with a limited energy source, and be inexpensive so they can be deployed in large numbers. The state of the art wireless sensor networks typically use conventional spread spectrum, or narrowband RF physical links. When WSN nodes are scattered in harsh RF environments, even the most advanced commercial of the shelf (COTS) radio will face significant problems at high data rate communications between the sensor nodes. In this paper we present an ultra-wideband (UWB) approach for inter-node communications in a wireless sensor network that is selforganized with respect to power efficiency, scalability, as well as robust to multipath and various types of interference. The organization of the rest of this paper is as follows. Section II discusses the key design challenges in wireless sensor networks. Section III introduces an UWB-based wireless sensor network and addresses its advantages and challenges. Section IV introduces the feedback loop TR receivers. Section V discusses the self-organization idea that can be achieved by feedback loop TR receivers in WSNs. Concluding remarks are summarized in Section VI.

This work was performed under the auspices of the U.S. Department of Energy by University of California Lawrence Livermore National Laboratory under contract No. W-7405-Eng-48.

\section{WIRELESS SENSOR NETWORK REQUIREMENTS}

Wireless sensor networks consist of a large number of sensor nodes that are spread in hardly accessible environments to detect a physical phenomenon and transfer the information through wireless links to the destination. Due to the dense deployment of sensor nodes in remote areas, the successful design of WSNs poses unique challenges and requirements that need to be addressed carefully. The main design challenges in WSNs can be categorized into the following areas:

- Scalability: As the number of sensor nodes in a wireless network increases, scalability imposes difficulties in transferring data. In order to send information to far away nodes, signals with higher transmission power should be employed which can cause inter-node interference or a multihop approach need to be considered.

- Power conservation: The nodes in wireless sensor networks have limited energy resources so to extend the lifetime of the entire network, power conservation in individual nodes is of significant importance. In WSNs, radio communications is the major consumer of energy. Hence, minimizing the radio transmission power or avoiding the unnecessary communications can considerably save power in sensor nodes.

- Synchronization: In radio communications between sensor nodes of a WSN, sensors continuously listen to transmissions and consume power if they are not time synchronized with each other. While global synchronization is unrealistic due to the large sensor population, node-by-node synchronization becomes a necessity in WSN design.

- Channel estimation: Channel estimation plays a critical role in WSNs, since sensor nodes communicate over wireless channels and have to overcome the effects of wireless link, such as noise, multipath effect, intentional jamming and internode interference. Estimating the wireless link between a specific transmitter and receiver pair provides directionality and reliable data transfer between the nodes.

- Self-organization: With the large number of sensor nodes deployed in remote environments, the ability of individual sensor nodes to self-organize is vital. Self-organization should be done in a way to improve the performance while reducing the power consumption of the entire sensor network.

With the increased popularity of wireless sensor networks in the last decade, there has been extensive research on the best way to approach each one of the above design issues both in hardware, and algorithmic level [1-2]. For radio 
communications and synchronization among the nodes, conventionally narrowband TDMA approach is suggested in most of the literature on wireless sensor networks. The strong narrowband signals used in conventional radio communications between the nodes create the problem of inter-node interference and results in unreliable transfer of information. Our goal in this paper is to introduce a UWB approach for radio communications among the sensor nodes in a WSN that addresses each one of the design challenges explained in this section.

\section{UltRA-WIDEBAND APPROACH TO WIRELESS SENSOR NETWORKS}

Unlike conventional RF communications, ultra-wideband transceivers use carrierless, short duration (pico second to nano second) pulses to transmit and receive information. The short duration of UWB pulses spreads their energy across a wide range of frequencies from near DC to several Gigahertz. This large bandwidth provides high capacity and low probability of detection properties for UWB communication systems. Using UWB technology for inter-node communication of WSNs, will not only offer small form factors, but will also provide high performance for communication over the wireless channels in spite of multipath distortions. Furthermore, transmission of short duration UWB pulses requires much lower power compared to strong narrowband signal transmission. In UWB-based WSNs, nodes can only communicate with their close-by neighbors due to low transmission power and avoid the inter-node interference issue that exists in narrowband techniques.

Despite all the benefits that UWB technology offers to the design of WSNs, it can create a unique set of challenges also. Employing the low powered UWB pulses for inter-node communications, introduces the scalability problem in WSNs. As the distance between nodes or the number of nodes increases, weak UWB pulses cannot transfer the information between the nodes properly. Also the short duration of UWB pulses introduces a major challenge in time synchronization for sensor nodes in a wireless network. In order to synchronize subnanosecond pulses, very high-speed ADC components are needed. Another problem with using UWB technology for wireless sensor networks is the performance degradation due to interference from strong narrowband signals that share the spectrum with low powered UWB pulses [5]. Moreover, detection of UWB pulses is commonly performed using classical matched filtering technique. Where the received signal is correlated with a UWB pulse template, thus wireless channel effects (such as multipath) on the received signal can significantly degrade the detection process due to low correlation between the predefined template and the distorted received signal.

The challenge posed by synchronization and channel degradation to narrow and low powered UWB pulses has been addressed by transmitted-reference (TR) modulation [3-5]. TR receivers correlate the received signal with a delayed replica of itself as shown in Fig.1.

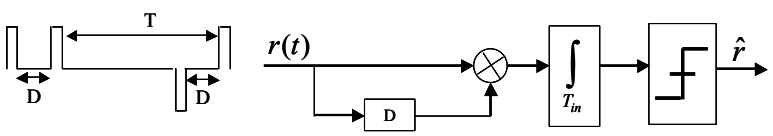

Figure 1: Block diagram of a TR receiver. Where $r(t)$ is the received signal, $D$ is the delay between the pulses in a TR doublet, $\mathrm{T}$ is the symbol repetition period, $\mathrm{T}_{\text {in }}$ is the finite integration window, and $\hat{r}$ is the decoded data.
In UWB-TR communication systems, data is modulated using a pair of pulses or doublets. The first pulse, "Tr", is modulated by data and is followed by an un-modulated "Rf" pulse, separated in time by " $D$ " interval. These receivers exploit multipath phenomenon to improve their performance in dense multipath channels. This way the wireless channel between transmitter/receiver pairs can be estimated for reliable data transfer. Furthermore, TR receivers are self-synchronized and eliminate the need for individual pulse synchronization with locally generated templates that exists in conventional pulse detection schemes [3-4].

Using UWB-TR transceivers for radio communication of WSNs offers simplicity, low transmission power, and capability to reduce the stringent UWB synchronization requirements, as well as channel estimation. On the other hand the performance of TR receivers is considerably limited by the severity of noiseon-noise component introduced by correlation of noise in " $\mathrm{Tr}$ " and "Rf" pulses in the received signal [5]. Therefore, scalability becomes a major issue in designing WSNs with UWB-TR transceivers. The noise-on-noise component can also decrease the accuracy of channel estimation in UWB-TR communication systems. In this paper we take advantage of benefits that TR receivers bring to WSNs and propose a new class of TR receivers to mitigate the noise-on-noise component in the received signal. The proposed receiver offers self-organization in WSNs by discovering the neighbors to communicate with through superior scalability, channel estimation while conserving communication power.

\section{FEEDBACK LoOP TR RECEIVERS}

In this section we introduce a simple and novel scheme to actively suppress interference in TR receivers while preserving the desired UWB signal and make TR receivers more suitable for use in WSNs. Interference suppression is achieved by introducing a feedback loop mechanism to enhance the "Rf" pulses in TR doublets. The block diagram of the proposed "Rf" enhancing feedback loop mechanism for TR receivers is shown in Fig. 2.

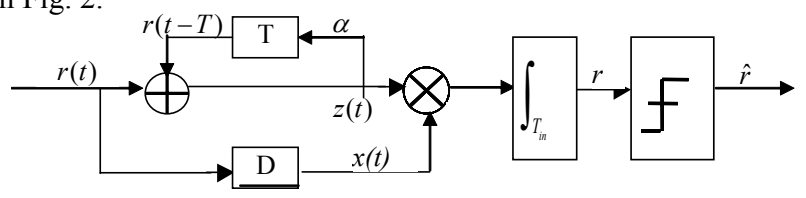

Figure 2: Block diagram of a modified TR receiver with "Rf" pulse enhancement feedback loop mechanism

The feedback loop contains an averaging delay, $T$, equal to the symbol repetition period (see Fig.1) and a loop loss factor $\alpha$ smaller than "1". As shown in Fig. 2, the received signal goes through multiple iterations around the feedback loop. With the loop delay, $T$, set to the symbol repetition period, the "Rf" pulses captured in each iteration will overlap and their signal power is enhanced each time. This mechanism works based on the assumption that the interference is uncorrelated with the signal, hence each circulation of input signal through the feedback loop makes "Rf" pulses cleaner by rejecting the interference. Please note that the feedback loop mechanism only improves the SNR of "Rf" pulses, since "Tr" pulses may have opposite polarity depending on the transmitted data, hence they do not experience the same resonance all the time. Fig. 3

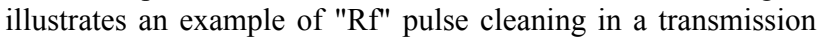
channel consisting of AWGN and multiple narrowband jamming signals. 

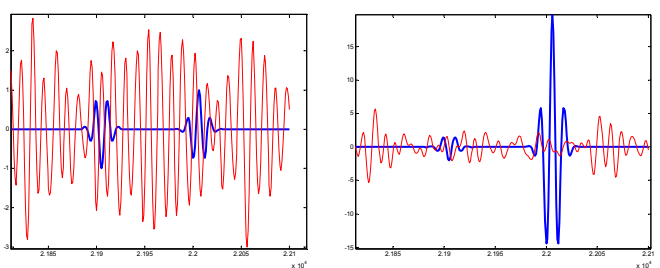

Figure 3: "RF" pulse cleaning in a transmission channel consisting of AWGN and NBI. (Left) An UWB TR doublet (solid lines) in the presence of strong interference (dotted lines). (Right) Cleaned "Rf" pulse after 100 loop iterations with $\alpha=0.95$.

\section{Self-Organization Technique}

In our proposed wireless sensor network, nodes communicate with their neighbors using UWB feedback loop TR (FBL-TR) transceivers. The short range of UWB devices makes them capable of establishing links with their close neighbors in a defined range. Therefore, several clusters of sensor nodes are formed based on the communications range of UWB transceivers. Using FBL-TR transceivers for radio communications of WSNs provides self-organization in terms discovering the neighbors to communicate with by channel estimation, scalability, as well as power conservation.

\section{A. Channel Estimation}

UWB-TR receivers are capable of estimating the channel between transmitter/receiver pairs. This characteristic makes them superior in performance to conventional matched filters. However the noise-on-noise component discussed in previous section can significantly impact the accuracy of channel estimation in such receivers. Mitigating the interference in the received signal by the feedback loop mechanism prior to signal detection in TR receivers improves the channel estimation considerably. Using the transmission block diagram of UWB sensor nodes in Fig. 4, we compare the channel estimation performance of classical matched filters, TR receivers, and FBL-TR receivers analytically and by Matlab simulations.

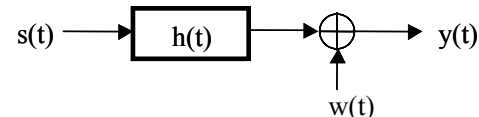

Figure 4: Transmission block diagram of sensor nodes

Where $s(t)$ represents the transmitted UWB signal, $h(t)$ is the multipath channel impulse response, $w(t)$ is additive white gaussian noise (AWGN), and $y(t)$ is the received signal given by

$$
y(t)=s(t) * h(t)+w(t)
$$

The '*' notation represents the convolution operation. The following equations show the UWB signal detection based on their receiver model.

Matched filter :

$\hat{r}=y(t) \oplus s(t)=[s(t) * h(t)+n(t)] \oplus s(t)$

TR receiver :

$\hat{r}=y(t) \oplus s(t)=[s(t) * h(t)+n(t)] \oplus[s(t) * h(t)+n(t)]$

$F B L-$ TR receiver :

$\hat{r}=y(t) \oplus s(t)=[s(t) * h(t)+n(t)] \oplus[s(t) * h(t)]$

Here $\oplus$ represents the correlation operation. As shown in (2), matched filter is expected to offer the worst correlation performance since the received signal has very low correlation with the original transmitted signal due to multipath channel effects. TR receivers are able to estimate the channel as shown in (3) since the received signal is correlated with a delayed version of itself. Therefore, the channel distortion on both signals will be the same that results in high correlation. However, the correlation of the noise components causes a limitation in the performance of those receivers. The best performance can be expected from the FBL-TR receivers in (4), where the channel is estimated and the correlation of noise components is removed. These results can be shown by the histogram of error in correlation process of the above receivers, shown in Fig.6.

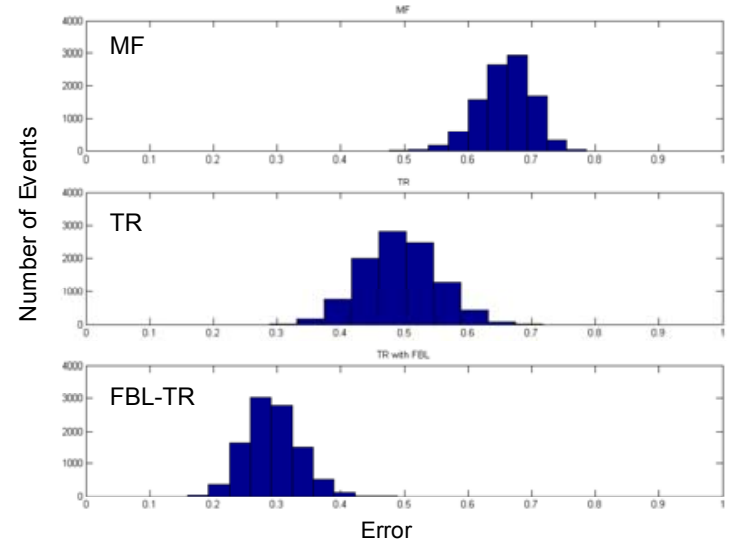

Figure 5: Histogram of error in correlation for matched filters, TR receivers, and feedback loop TR receivers. Maximum error is normalized to 1 .

This figure demonstrates the results that was expected from (2), (3), and (4). Matched filters demonstrate the worst performance with maximum error being concentrated around 0.7. In TR receiver the maximum error is concentrated around 0.5 and as expected from the equations the FBL-TR receiver shows the best performance of all by having the maximum error centered on 0.25 .

\section{B. Scalability:}

As mentioned earlier, wireless sensor networks need to be scalable with respect to change in network size, node density and node topology. Some nodes may die over time and therefore, the remaining nodes should be able to establish links with nodes in a larger distance. The noise-on-noise component in UWB-TR receivers [4] can severely limit the range of the sensors communication system and directly impact the networks scalability attribute.

Adding the feedback loop mechanism to UWB-TR receivers, mitigates the interference that exists in "Tr" and "Rf" pulses and strengthens the UWB signal to improve the communication range and scalability. The following derivations provide the SNR of a FBL-TR receiver. From Fig. 2, $r$ is given by

$$
r=\int_{T_{i n}} z(t) x(t) d t
$$

After $n$ iterations around the feedback loop, the signal and noise components of $z(t)$ are scaled by $n$, while $x(t)$ remains untouched as

$$
r=\int_{T_{\text {in }}}[n s(t)+\sqrt{n} w(t)][s(t)+v(t)] d t
$$

where $w(t)$ and $v(t)$ are different samples of AWGN with $\mathrm{N}_{0} / 2$ double-sided power spectral density, independent and 
uncorrelated with each other. The signals going through $n$ loop iterations are added coherently $n$ times, while noise components at the output of feedback loop add incoherently with standard deviation of $\sqrt{n}$. The multiplication in (6) results in the following 4 integrals:

$$
\begin{array}{r}
r=\int_{T_{i n}}[n s(t) s(t)] d t+\int_{T_{i n}}[\sqrt{n} w(t) s(t)] d t+ \\
\int_{T_{i n}}[n s(t) v(t)] d t+\int_{T_{i n}}[\sqrt{n} w(t) v(t)] d t
\end{array}
$$

The first integral provides the signal component $\left(n E_{P}\right)$, the second and third integrals represent signal-on-noise interference $\left(I_{S-N}\right)$, and finally the fourth term represents the noise-on-noise interference $\left(I_{N-N}\right)$. Therefore, the receiver's SNR can be calculated as

$$
S N R=\frac{n^{2} E_{P}{ }^{2}}{\operatorname{Var}\left(I_{S-N}+I_{N-N}\right)}=\frac{n^{2} E_{P}{ }^{2}}{E\left\{\left(I_{S-N}+I_{S-N}\right)^{2}\right\}}
$$

In order to find the noise energy, the variance of the total interference should be calculated as

$$
\begin{aligned}
& \left(I_{S-N}+I_{N-N}\right)^{2}= \\
& {\left[\int_{T_{i n}} \sqrt{n} w\left(t_{1}\right) s\left(t_{1}\right) d t_{1}+\int_{T_{i n}} n s\left(t_{1}\right) v\left(t_{1}\right) d t_{1}+\int_{T_{i n}} \sqrt{n} w\left(t_{1}\right) v\left(t_{1}\right) d t_{1}\right] .} \\
& {\left[\int_{T_{i n}} \sqrt{n} w\left(t_{2}\right) s\left(t_{2}\right) d t_{2}+\int_{T_{i n}} n s\left(t_{2}\right) v\left(t_{2}\right) d t_{2}+\int_{T_{i n}} \sqrt{n} w\left(t_{2}\right) v\left(t_{2}\right) d t_{2}\right]}
\end{aligned}
$$

Using the same approach as [4], the variance of total interference is given by

$$
E\left\{\left(I_{S-N}+I_{N-N}\right)^{2}\right\}=\frac{n E_{P} N_{0}}{2}+\frac{n^{2} E_{P} N_{0}}{2}+\frac{n T_{i n} B N_{0}{ }^{2}}{4}
$$

where $T_{i n} B$ is the time-bandwidth product of the UWB pulse. Therefore, the receiver's SNR can be calculated as

$$
S N R=\frac{2 n^{2} E_{p}{ }^{2}}{E_{P} N_{0}\left(n+n^{2}\right)+\frac{n T_{i n} B N_{0}{ }^{2}}{2}}
$$

Considering the input SNR to be $S N R_{i n}=E_{P} / N_{0}$, the receiver's $\mathrm{SNR}$ with respect to $\mathrm{SNR}_{\text {in }}$ can be represented as

$$
S N R=\frac{2 n^{2}}{\frac{\left(n+n^{2}\right)}{S N R_{i n}}+\frac{n T_{i n} B}{2\left(S N R_{i n}\right)^{2}}}
$$

At low input SNRs the second term in the denominator becomes the dominating interference factor. Therefore, the receiver's SNR can be approximated as

$$
S N R \cong \frac{2 n^{2}}{\frac{n T_{i n} B}{2\left(S N R_{i n}\right)^{2}}}=\frac{4 n\left(S N R_{i n}\right)^{2}}{T_{i n} B}
$$

Equation (14) shows that the FBL-TR receiver's output SNR is increased by $4 n\left(S N R_{i n}\right)^{2} / T_{i n} B$. This equation reveals that the receiver is capable of decoding the incoming signals with much lower signal-to-noise ratios. The ability to process the Low $S N R_{\text {in }}$ to achieve the required SNR for a successful communication link, leads to an improved sensor node range. This result is noted by the relationship between the communications range, $R$, transmit power $P_{T}$ and input SNR described in [6] as

$$
R^{2} \propto \frac{P_{T}}{S N R_{i n}}
$$

Assuming constant $P_{T}$, low $\mathrm{SNR}_{\text {in }}$ directly relates to improved range. The enhanced range for sensor nodes is needed for the scenarios where some neighboring nodes die due to lack of power, so information bearing nodes are required to establish communications link with other nodes in a longer distance away from their normal communications range.

\section{Network Power Conservation}

Although UWB transmitters in WSNs use low energy to transmit short duration pulses, continuous listening for information on the receiver side can still consume a large amount of energy. Therefore, limiting unnecessary listening can provide power saving advantages to each sensor node and consequently to the entire network.

In FBL-TR receivers, the backend detection part of the receiver can be shut down while leaving the front-end interference mitigation feedback loop activated. The feedback loop mechanism continuously enhances the SNR of the received signal until the received signals' interference is suppressed. Once the received signal reaches a specific threshold and is strong enough for reliable communications, the TR receivers will be activated and detect the received bits.

This form of self-organization provides considerable power saving for individual nodes as well as the entire circuit. Since the feedback loop is a passive circuit and can run continuously in each sensor while using minimal amount of power. In this manner every node whose received signal has reached the assigned threshold, is self-organized to activate its receiver.

\section{Conclusions}

In this paper we proposed a UWB based approach for inter-node communications of wireless sensor networks. The UWB receivers use a feedback loop mechanism in addition to TR modulation to detect the data. The feedback loop mechanism offers self-organization by mitigating the inter-node interference, scalability, power conservation, and channel estimation to the individual sensor nodes in a wireless sensor network.

\section{REFERENCES}

[1] G. Asada, M. Dong, T.S. Lin, F. Newberg, G. Pottie, W.J. Kaiser, and H.O. Marcy "Wireless Integrated Network Sensors: Low Power Systems on a Chip". Proceedings of the 1998 European Solid State Circuits Conference.

[2] A. Bhatnagar and T. Robertazzi, "Layer Net: A New SelfOrganizing Network Protocol,” IEEE MILCOM 90 Conf. Record, 1990, pp.845-849.

[3] R. Hoctor, H. Tomlinson, "Delay-hopped Transmitted-Reference RF Communications," 2002 IEEE Conference on Ultra Wideband Systems and Technologies, May 2002.

[4] A. Spiridon, F. Dowla, F. Nekoogar, D. Benzel, " On the performance of ultra-wideband transmitted-reference receivers", IEEE Trans. Comm. (submitted to publication.)

[5] F. Dowla, F. Nekoogar, F. Spiridon, "Interference Mitigation in Transmitted-Reference Ultra-Wideband Receivers", IEEE International Symposium on Antennas and Propagation (APS 2004), Monterey, CA June 20-26, 2004.

[6] R.J. Fontana, On "Range-Bandwidth per Joule" for Ultra Wideband and Spread Spectrum Waveforms", http://www.multispectral.com/pdf/UWB DSSS.pdf 